\title{
Une approche instrumentale de la transposition didactique
}

Quelques thèses illustrées par l'analyse de l'enseignement de quelques objets de la discipline « français »

An instrumental approach of didactic transposition. Some theses illustrated by the analysis of some objects taught in the subject matter French

Bernard Schneuwly et Christophe Ronveaux

\section{(Q) OpenEdition \\ Journals}

\section{Édition électronique}

URL : https://journals.openedition.org/pratiques/9515

DOI : $10.4000 /$ pratiques.9515

ISSN : 2425-2042

\section{Éditeur}

Centre de recherche sur les médiations (CREM)

\section{Référence électronique}

Bernard Schneuwly et Christophe Ronveaux, « Une approche instrumentale de la transposition didactique », Pratiques [En ligne], 189-190 | 2021, mis en ligne le 07 juillet 2021, consulté le 23 juillet 2021. URL : http://journals.openedition.org/pratiques/9515; DOI : https://doi.org/10.4000/pratiques. 9515

Ce document a été généré automatiquement le 23 juillet 2021.

(c) Tous droits réservés 


\section{Une approche instrumentale de la transposition didactique}

Quelques thèses illustrées par l'analyse de l'enseignement de quelques objets de la discipline « français »

An instrumental approach of didactic transposition. Some theses illustrated by the analysis of some objects taught in the subject matter French

Bernard Schneuwly et Christophe Ronveaux

« Mais le nouveau procède de l'ancien et en est le degré suivant. Nous cherchons moins à réaliser quelque chose de tout différent, hors de tout chemin tracé, qu'à franchir le pas suivant, c'est-àdire à tirer la conclusion de ce qui est. On fait du neuf en bouleversant l'ancien, en le continuant, en le développant. » (Brecht, 1979, p. 150)

\section{Introduction}

S'insérant dans le projet du présent numéro spécial de Pratiques, qui «vise à faire un point épistémologique [...] sur les concepts en usage », dans cette contribution nous explicitons notre usage du concept de " transposition didactique » au cœur de notre projet de recherche depuis une trentaine d'années (Schneuwly, 1995 ; Bronckart \& Plazaola Giger, 19981). Cet usage est particulier, dans la mesure où nous donnons à ce concept une signification qui s'inscrit dans la continuité des travaux didactiques inauguraux, mais le transformons en l'arrimant à une approche instrumentale. Cette dernière est ancrée dans une autre œuvre théorique, celle de L.S. Vygotskij ${ }^{2}$, qui, depuis presque quarante ans (Schneuwly \& Bronckart, 19853), est à la base de nos travaux d'élaboration collaborative de séquences didactiques pour l'enseignement de la production de textes oraux et écrits (Bain et al. 1985 ; 1988). Au cœur de sa conception du développement de la personne, le chercheur russe définit la médiation comme l'appropriation d'œuvres humaines matérialisées dans des signes par le passage de 
l'interpsychique à l'intrapsychique ${ }^{4}$. L'articulation de ces deux constructions théoriques ne va pas de soi. Faut-il marquer les différences, voire les contradictions, et exacerber le débat? Ou favoriser le greffon en soulignant les limites et les vertus de l'emprunt? Dans la présente contribution, plutôt que de renvoyer ces constructions théoriques dos à dos en posant leurs possibles contradictions ${ }^{5}$ et de rendre compte, pour chacune de nos thèses, de l'immense littérature publiée à ce jour sur la transposition didactique, nous avons choisi de montrer quelques-unes des solutions théoriques élaborées à partir de notre travail empirique sur le fonctionnement de l'enseignement dans la discipline «français ». Nous renvoyons en note quelques-uns des travaux fondateurs. Il n'échappera à personne que ce travail empirique s'inspire profondément de l'approche instrumentale dans la manière de définir son objet de recherche, sa méthodologie, sa méthode de recueil des données et son modèle d'analyse.

2 Dans la perspective que nous venons de décrire, il s'agit dès lors de définir quelques concepts que nous avons forgés tout au long de notre programme de recherche pour interpréter les observations et mener les analyses. La forme textuelle qui nous parait la plus adéquate pour réaliser ce projet est celle de sept thèses articulées les unes aux autres, présentant les concepts dont nous justifions l'usage et l'utilité par quelques résultats de nos recherches empiriques. L'enchainement de l'exposé de ces thèses n'est pas fortuit : il reproduit peu ou prou la logique des différents états du questionnement de vingt années de recherche.

\section{Thèse 1 : la transposition didactique est l'objet de recherche central de la didactique}

3 L'une des manières de concevoir la transposition didactique est la suivante $^{6}:$ si l'on définit le didactique, objet de la didactique, comme la transmission et diffusion de savoirs et de pratiques, d'ailleurs consubstantiellement liés, dans des institutions spécialisées à cet effet, la transposition didactique en est constitutive, avec le système didactique. Les savoirs et pratiques sont en effet transportés de là où ils sont utilisés vers une institution où ils deviennent objets d'enseignement et d'apprentissage ${ }^{7}$. Ainsi prennent- Ils un autre sens, dans un processus souvent désigné par désyncrétisation et décrit par un mouvement de décontextualisation et recontextualisation. Ils sont également modélisés pour les rendre enseignables et apprenables, décomposés en éléments et mis en ordre selon une progression ${ }^{8}$. Ces décompositions et leur organisation progressive dépendent de la nature des savoirs mais aussi des pratiques et des institutions.

4 Classiquement, le processus de transposition est présenté en deux volets : externe et interne. La dimension externe a trait aux rapports multiples et sans cesse changeants entre les lieux d'usage des savoirs et leur transport dans l'institution d'enseignement et de formation, l'institution elle-même ayant une autonomie importante de traitement, voire de production de savoirs à des fins d'enseignement créant une véritable « culture scolaire " (Denizot, 2018). Il n'en reste pas moins qu'il y a toujours, en dernière instance, un rapport entre savoirs et savoir-faire à enseigner d'une part, savoirs de référence extérieurs à l'école d'autre part. Nous reviendrons sur cette problématique à travers le concept de disciplinarisation (thèse 8). 
Dans la transposition interne, «l'enseignant est le servant de la machine didactique dont le moteur est la contradiction entre ancien et nouveau " (Chevallard, 1985, p. 71). Il s'agit en effet de transformer l'objet à enseigner en objet enseigné. Cette contradiction est mise en œuvre par un temps d'enseignement qui repose sur cette fiction : l'élève suit pas à pas la construction d'un objet d'enseignement élémenté par un apprêt didactique prévu pour que cet objet puisse être approprié. C'est ici qu'il nous parait possible d'articuler la théorie de la transposition didactique avec la conception vygotskienne du rapport entre enseignement et développement. Conscient lui aussi de la contradiction fondatrice de l'enseignement, L. S. Vygotskij note qu' " il serait faux de croire que, si, dans ce semestre, l'élève suit l'enseignement en quelque chose en arithmétique, il fera par conséquent au cours de ce même semestre les mêmes progrès dans son développement interne" (Vygotskij, 1985 [1934], p. 266). L'élève, tout en suivant la fiction du dépliage pas-à-pas de l'objet d'enseignement, construit lui-même ses capacités selon une logique propre. Autrement dit, il doit faire ce qu'il ne sait pas (encore) faire grâce à la mise en scène didactique par des dispositifs qui se suivent et sa régulation continuelle ${ }^{9}$. Cette dernière est nécessaire à cause des problèmes complexes de prise en compte de l'ancien, préconstruit, supposé connu, et des modalités de transformation de l'ancien qui, précisément, ne correspondent pas nécessairement au temps de l'enseignement. L'objet enseigné apparait dès lors sans cesse changeant, jamais fini, comme un tissage continuel de significations nouvelles par transformation et enrichissement d'anciennes. Ce processus potentiellement illimité est pourtant organisé avec un début où l'objet d'enseignement est introduit et une fin - le bornage délimite la séquence - ayant pour résultat un objet enseigné, produit du processus où se rencontrent enseignement d'une part, apprentissage et développement de l'autre, chacun suivant sa logique.

Cette manière de définir la transposition didactique interne comme objet de recherche didactique nous amène à poser deux questions auxquelles nous tentons de donner des réponses : de quels outils ou instruments ${ }^{10}$ le «servant » dispose-t-il pour mettre en marche la «machine didactique», pour reprendre la métaphore chevallardienne ? Et comment organise-t-il le temps pour mettre en œuvre cette machine?

\section{Thèse 2 : le travail enseignant repose sur des outils sémiotiques pour transformer les modes de penser, de dire et de faire des élèves ${ }^{11}$}

7 Le travail de l'enseignant, comme tout travail, nécessite des moyens :

Le moyen de travail est une chose ou un complexe de choses que le travailleur introduit entre lui-même et l'objet du travail et qui lui sert de conducteur effectif de son activité sur cet objet. Il utilise les propriétés mécaniques, physiques et chimiques des choses, pour le laisser agir comme moyens de pouvoir sur d'autres choses, selon les fins qu'il vise. (Marx, 1953, p. 208 ; traduction de B. Schneuwly).

8 Cependant, contrairement à l'objet du travail de l'ouvrier, " l'objet » du travail enseignant, à savoir les modes de penser, dire et faire des élèves, ne peuvent être «travaillés » directement. C'est ce que montre bien la différence fondamentale décrite plus haut entre processus d'enseignement d'une part, d'apprentissage et de développement de l'autre, nécessairement séparés. Le travail de l'enseignement a cette fonction d'introduire du «nouveau» qu'il doit articuler à l'ancien selon une 
temporalité qui est celle d'un avancement graduel régulé par la présentation et l'élémentation de l'objet d'enseignement. Cette temporalité graduelle a pour effet les transformations qui s'opèrent du côté des élèves, ou plutôt, les transformations qu'opèrent les élèves sur eux-mêmes. Cet effet repose sur deux formes de médiations imbriquées que la conceptualisation de L.S. Vygotskij permet d'expliciter. L'idée fondamentale est la suivante :

Les outils psychologiques sont des formations artificielles; ils sont par nature d'essence sociale, et ne constituent pas des adaptations organiques ni individuelles; ils sont orientés vers la maitrise des processus - chez autrui aussi bien que chez soimême, comme la technique l'est vers celle des processus de la nature. (Vygotskij, 2014 [1930], p. 567)

9 Ces outils psychologiques sont les signes ou ensembles de signes de différents types, autrement dit des outils sémiotiques. «Le rôle de l'outil dans une opération de travail est analogue à celle du signe dans le comportement : la fonction instrumentale ${ }^{12}$ » (2014 [1928-1930], p. 203). La particularité du travail enseignant réside dans le fait qu'il y a, certes, «maitrise des processus [...] des autres ", mais une maitrise qui est loin d'être celle des outils techniques. On peut le décrire en ces mots : l'enseignant introduit des dispositifs ${ }^{13}$ qui impliquent une double sémiotisation de l'objet d'enseignement, première forme de médiation. D'une part, ce dernier doit être « rendu présent » sous des formes diverses en tant qu'objet à étudier, ce qui n'est possible qu'imparfaitement, puisque la connaissance de l'objet est précisément le but. D'autre part, les différentes caractéristiques de l'objet doivent être mises en évidence, pointées, verbalisées, questionnées, problématisées. Ici apparait la deuxième forme de médiation : la constitution de l'objet d'étude et sa décomposition et problématisation implique l'interaction entre enseignant et élèves, condition de la transformation des modes de penser, parler et faire des élèves. Ils constituent les outils sémiotiques médiatisant cette possible transformation. On retrouve ici une autre dimension fondamentale de la théorie de L.S. Vygotskij : le passage de l'inter- à l'intrapsychique, le processus d'intériorisation comme mécanisme de base de la construction de fonctions psychiques nouvelles, la capacité d'écriture par exemple, comme synthèse de nombreuses autres fonctions.

Dans notre programme de recherche, nous avons défini deux unités méthodologiques pour observer et analyser la médiation par les dispositifs, considérés comme outils ou instruments de l'enseignement, à deux niveaux : celui de l'objet d'enseignement comme un tout, la séquence (thèse 3 ), et sa décomposition en une chaine de dispositifs spécifiques d'une discipline, proche de ce que Y.Chevallard (1992, p.95) a proposé d'appeler la «mésogenèse » (thèse 4).

\section{Thèse 3 : La séquence d'enseignement est l'unité d'observation du travail de l'enseignant et de l'objet enseigné.}

11 La séquence d'enseignement, définie comme une suite de dispositifs didactiques structurés dans le temps et hiérarchisés, est chapeautée par un objet d'enseignement qui fait son unité (Cordeiro, Ronveaux \& Grafe, 2009, p. 93 sq.). Même si une séquence s'effectue, en règle générale, selon un même plan pour toute une classe, des activités diversifiées, réalisées en parallèle, se déroulant dans des lieux différents (notamment à 
la maison), sont possibles. C'est l'objet d'enseignement, en général commun, qui fait la cohérence de la séquence d'enseignement. Il correspond en général à un objet prescrit par un plan d'étude ou un programme ou des instructions officielles. Il est le point de départ des nombreuses séquences d'enseignement du français que nous avons analysées, concernant par exemple la phrase relative, la production d'un texte d'opinion, la lecture (littéraire) de Candide, celle du Loup et de l'agneau, les discours d'escorte de textes littéraires, les métatextes d'auteurs, la lecture de témoignages et de récits de la Première Guerre mondiale.

La séquence d'enseignement constitue l'unité d'observation privilégiée pour saisir ce qui s'enseigne, à savoir la transposition didactique interne, pour deux raisons :

(i) elle donne au chercheur un instrument de découpage et de structuration de

l'objet enseigné dans le flux des interactions scolaires ;

(ii) elle permet la comparaison de pratiques plurielles qui lui apparaissent souvent dans des variations interindividuelles et contextuelles extrêmes qui rendent difficiles la modélisation.

La séquence, dont l'enseignant marque plus ou moins les contours (un début et une fin), détermine, pour le chercheur, un point de vue institutionnel sur l'objet. L'enseignant est le principal maitre d'œuvre qui délimite l'objet et le rend saisissable par les élèves au moyen de dispositifs divers puisés dans la panoplie élaborée par la profession durant la longue histoire d'une discipline scolaire.

Dans le processus de recherche, afin de rendre intelligibles l'évolution de l'objet enseigné - le processus de transposition didactique interne-, la séquence d'enseignement, enregistrée et transcrite, est transformée en synopsis ${ }^{14}$. Dans cette opération de condensation, le chercheur restitue le dépliage de l'objet dans le temps, en identifiant à la fois les éléments de l'objet et les liens hiérarchiques que les éléments de l'objet entretiennent dans la séquence. Le travail d'interprétation mené pour élaborer le synopsis suppose de reconstituer l'élémentation de l'objet d'enseignement opérée par l'enseignant. Cette reconstitution doit pouvoir rendre compte des diverses tensions qui mettent en mouvement l'objet enseigné, tensions générées avant tout par la confrontation des élèves à l'objet. Le texte du synopsis représente une première analyse, mais fait partie intégrante du corpus à analyser. C'est la collection des synopsis qui permet la comparaison des objets enseignés dans différentes classes, éventuellement à différents niveaux scolaires. Le changement d'échelle que constitue le synopsis donne à voir, par la comparaison des séquences, des phénomènes didactiques nouveaux.

Prenons l'exemple de l'objet d'enseignement prescrit par le plan d'études romand, la production d'un texte d'opinion. De la comparaison, le chercheur dégage des trames prototypiques qui modélisent l'objet enseigné dans le mouvement de sa construction. Ainsi, l'on a pu identifier trois modalités de construction de l'objet (Schneuwly \& Dolz, 2009, p. 222 sq), qui correspondent peu ou prou aux trames prototypiques suivantes :

(i) une trame centrée sur l'unité du texte d'auteur, pour laquelle chaque texte correspond à une séquence ;

(ii) une trame centrée sur l'unité générique du texte d'opinion, qui comprend une structure introductive et conclusive de production et une séquentialité modulaire entre ce début et cette fin ;

(iii) une trame centrée sur l'unité du genre scolaire de la dissertation, qui s'ouvre sur un corpus de textes décrivant des situations argumentatives et se poursuit par des exercices de repérages d'arguments dans des textes variés. 
16 et des objets enseignés, et de mieux comprendre la détermination des modèles didactiques de la discipline et conceptions de l'enseignant sur le processus de transformation des objets de savoirs (thèse 6).

Se pose alors la question du rapport des éléments de la séquence à l'objet enseigné. D'une part, la somme des éléments distribués dans une séquence d'enseignement ne fait pas la totalité de cet objet. D'autre part, un même objet à enseigner peut être décomposé en éléments divers qui relèvent de modèles distincts, lesquels éléments sont distribués dans des séquentialités variées. La comparaison de ces unités d'enseignement que sont les séquences permet de rendre compte d'un des aspects caractéristiques du processus transpositif, la distribution dans le temps d'éléments d'enseignement selon la logique graduelle de dispositifs sans cesse confrontés au collectif de la classe. Par comparaison, on voit aussi que la variation des séquences n'est pas seulement déterminée par des facteurs stylistiques interindividuels, mais aussi par des facteurs culturels liés aux dispositifs et aux objets de savoirs d'une discipline et de son histoire (thèse 5).

\section{Thèse 4 : la séquence d'enseignement, comme panoplie de dispositifs, est le « macro-instrument » de l'enseignant.}

Les séquences d'enseignement sont élaborées par les enseignants à partir d'une panoplie de dispositifs qui constituent autant d'instruments de médiation facilitant l'appropriation de l'objet d'enseignement par les élèves. Elles peuvent aussi être la mise en œuvre de séquences préconstruites ou co-construites, fruits d'ingénieries didactiques. En cela, elles constituent un "macro-instrument» que s'approprie l'enseignant. La panoplie des dispositifs mis en œuvre constituent l'une des dimensions de la transposition didactique interne.

Dans son travail de thèse sur l'appropriation d'une séquence didactique sur le résumé informatif, M. Wirthner (2017) montre comment des enseignants transforment un macro-instrument proposé par le chercheur selon leurs conceptions au moyen de leurs propres instruments, mais aussi comment ce macro-instrument les transforme dans leurs conceptions, dans leur manière d'enseigner le texte, dans leur style d'enseignement. Cette recherche met aussi en évidence que les pratiques proviennent de couches historiques diverses: une voie très féconde aujourd'hui pour saisir les potentialités transformatrices de l'instrument et décrire le mouvement transpositif interne constitutif des activités de la transmission. Nous y reviendrons dans la suite du texte.

Les instruments didactiques dont l'enseignant dispose et qu'il active dans une situation donnée sont autant de dispositifs par lesquels il met en scène l'objet à enseigner. Un dispositif comprend autant les formes matérielles qui "scénographient» l'objet (manuel d'exercices imprimés ou consignes orales, tableau noir ou écran de projection, objet-livres ou photocopies d'extraits de textes, support papier ou numérique, organisation spatiale du travail, etc.) que les consignes et les modalités de traitement (questionnaire, lecture à voix haute individuelle ou chorale, résumé, lecture analytique, commentaire composé, contextualisation, écriture d'invention, réécriture, etc.) 
(Schneuwly, 2009). Le relevé de ces instruments et la description de leurs fonctions dans la séquence sont des analyseurs particulièrement féconds du processus transpositif. Dans le domaine de la lecture, S. Aeby Daghé $(2010,2014)$ a montré que ces dispositifs mis en œuvre par les enseignants correspondent à des familles de modalités. Le regroupement de ces dispositifs de lecture en genres d'activités scolaires permet de dégager de la très grande diversité des dispositifs inventoriés dans nos empiries une certaine systématique sociohistorique de la transposition. Elle montre que la panoplie de ces dispositifs et leur organisation sont circonscrites par la discipline et son histoire, par le niveau des élèves, par les classes d'objets à traiter. Cette catégorisation, appliquée au corpus de notre recherche sur le fait littéraire par exemple, a permis de mettre en évidence la place centrale du commentaire de texte comme instrument de médiation, malgré la très grande variété des dispositifs (Aeby Daghé \& Schneuwly, 2018). Toutefois, les démarches interprétatives, sollicitées par la nouvelle d'un auteur suisse contemporain, considéré par les enseignants comme « illisible », «bizarre », etc., interrogent cet instrument du commentaire et poussent les élèves et les enseignants à expérimenter de nouveaux dispositifs ou à recycler des dispositifs anciens, et à créer de nouvelles normes (thèse 6).

21 Il faut faire un pas de plus dans cette thèse sur les instruments de l'enseignant en posant que les dispositifs sont conditionnés à l'archiélève. Ce dernier ne renvoie pas seulement une entité abstraite et idéelle, sorte d'élève supposé une fois pour toutes qu'il suffirait d'interpeller dans la situation. Il est une aide à la décision au moment de planifier. En effet, au moment de sa réalisation, le macro-instrument de la séquence est transformé par les élèves, qui agissent sur les dispositifs, les questionnent, les mettent en cause et s'approprient l'objet enseigné, en perpétuel mouvement. Ces élèves transformateurs de dispositifs ne sont pas pour autant des sujets autonomes, cognitivement compétents, dégagés des effets du format et des dispositifs de la séquence. Ils interviennent au contraire, parce qu'ils ont intégré certains instruments, en poussant les usages des instruments jusqu'à leurs limites. O. Franck $(2017,2018)$ montre cette dialectique entre dispositifs prédéterminés par l'archiélève et modifiés par les élèves en situation. Comparant les séquences imaginées par les enseignants et les séquences réalisées à partir d'un même texte à différents degrés, elle met en évidence le travail conjugué des enseignants et des élèves sur les dispositifs. Par rapport aux dispositifs prévus, les écarts constatés attestent que des élèves interviennent sur les dispositifs en profondeur, créant souvent la surprise, notamment quand il s'agit d'objets d'enseignement peu assurés par une pratique didactique établie comme par exemple la lecture d'un texte littéraire contemporain dont la difficulté est davantage fonction d'une absence d'instruments didactiques pour le traiter en classe.

Une autre manière d'utiliser la séquence d'enseignement comme unité de recherche est d'observer les effets différentiels des filières scolaires créant de claires distinctions inégales entre groupes d'élèves. B. Védrines (2017) met ainsi en évidence les effets différentiels de l'organisation du système scolaire en filières sur le développement intellectuel des élèves. Contrastant les dispositifs mis en œuvre et leur enchainement, dans une filière préprofessionnelle et une autre menant à l'université, il constate que l'homogénéisation des classes scolaire en fonction des capacités construites d'abstraction induit la projection d'un archiélève et l'organisation de séquences d'enseignement qui lui correspondent - l'une des dimensions de la transposition didactique interne. Ceci provoque en retour l'homogénéisation régulière du niveau par la sélection et un positionnement différent des élèves dans la zone de développement 
proche qui, elle-même produit un niveau qui, etc. Toutes ces empiries montrent aussi l'importance que prend le texte dans la panoplie des dispositifs, moins par sa nature que par les usages qui en sont fait dans la classe. Dans nos dispositifs de recherche semi-expérimentaux, c'est cette perméabilité du texte aux usages qui fait de ce dernier un réactif particulièrement intéressant pour établir les variations diachroniques des pratiques.

\section{Thèse 5 : les pratiques d'enseignement sont toujours des sédimentations de différentes couches historiques de pratiques}

classe: les séquences d'enseignement se réfèrent à des démarches et dispositifs provenant de moments historiques différents, des plus classiques au plus contemporains. Nous référant à la métaphore de "structure géologique $»^{15}$ de la personnalité que L.S. Vygotskij (2014 [1928-1930], p. 169) utilise pour définir une méthode d'analyse de l'histoire du développement des fonctions psychiques supérieures, il nous parait possible de l'utiliser pour décrire les pratiques d'enseignement. En effet, ces dernières se forment à travers la rencontre des enseignants avec de nombreuses pratiques appartenant à des moments historiques différents : dans leur propre biographie d'élève, à différents niveaux d'une scolarisation longue et avec une très grande variété de personnes, dans les discours de la profession, dans les prescriptions et recommandations. Saisir le mouvement transpositif, sans aplatir cette épaisseur historique, en restituant ce qui, dans cette histoire, continue d'agir sur le processus, présuppose donc un travail sur de multiples sources : plan d'études, manuels, écrits pédagogiques, récits biographiques ${ }^{16}$. Cette connaissance permet de dégager dans les variations de pratiques en synchronie les traces de leurs transformations diachroniques. La continuelle mise en perspective des deux constitue l'une des démarches fondamentales dans notre travail d'analyse des pratiques; elle a permis de mettre en évidence de multiples formes de sédimentations, dépendant d'ailleurs des objets de l'enseignement.

Plusieurs de nos analyses attestent de ces formes variées sédimentées. L'une d'entre elles, celle qui compare les formes de sédimentation observées dans l'enseignement de la production de textes argumentatifs et dans l'enseignement de la phrase relative à des élèves de 14 ans, nous a conduit à interpréter les résultats sous la forme de paradoxe : la transformation structurelle de l'enseignement de la rédaction de textes agit en profondeur sur les pratiques et a pour effet des trames de séquences d'enseignement fortement contrastées (nous les avons présentées brièvement ci-dessus dans la thèse 3 ) ; la transformation radicale des notions constitutives de l'enseignement de la grammaire a des effets très visibles sur la terminologie notamment, mais ne transforme pas la trame constitutive de l'enseignement (Schneuwly \& Dolz, 2009, p.319 et suivantes).

Dans une autre analyse, celle sur les pratiques d'enseignement de la lecture de textes littéraires, nous décrivons l'évolution d'un des dispositifs les plus fréquents figurant dans nos empiries, le résumé. Cette description permet de voir la structure multicouche - complémentaire et non pas contradictoire - de la pratique enseignante (Ronveaux \&

Pratiques, 189-190 | 2021 
Schneuwly, 2018, p. 257 et suivantes). Le caractère "contemporain » du texte de l'auteur suisse, sans tradition didactique aucune, par contraste avec la fable de J. de La Fontaine, pousse enseignants et élèves à développer une activité de condensation qui se trouve peu utilisée dans la découverte du texte présenté comme « classique ». Dans le traitement du texte contemporain, se fait jour la nécessité de résumer le texte, soit sur l'invitation de l'enseignant qui prévoit peu ou prou une activité de synthèse, soit sous la pression des élèves qui réclament un résumé parce qu'ils n'ont « rien compris ». Il y a bien une double détente dans le processus de construction du sens, mais ce dernier ne correspond pas au modèle hiérarchisé d'une interprétation de premier et de deuxième degré, ni au modèle interprétatif de la hauteur, ni à celui des profondeurs. Tout se passe comme si les enseignants admettaient l'existence d'un premier degré, héritage d'une certaine tradition scolaire des lectures par degrés (qu'ils nomment en ces termes d'ailleurs), mais dont la fonction apparait dans une forme sédimentée, méthodologique seulement, sans nécessité cognitive de se rappeler pour mémoire. Dans nos empiries, l'instrument du résumé répond plutôt aux contraintes de la situation didactique d'appréhender un texte nouveau, de "se familiariser » avec lui, de " s'en imprégner », selon les termes des enseignants. La visée est proche de cet instrument multiséculaire décrit par A. Chervel (2006, p.609-610), l'analyse. Mais le contexte d'un récit intrigant contemporain et la place accordée au lecteur dans son appropriation transforment le résumé en instrument synthétique et structurant dont la visée est de s'accorder provisoirement sur l'enchainement des faits. Cet instrument synthétique vise moins à vérifier la compréhension qu'à valider auprès du collectif une hypothèse de scénario, forçant à faire converger la tension narrative de l'intrigue, alors que, précisément, celle-ci invite au jeu des divergences. De toute évidence, dans la synchronie de la classe se superposent des couches historiques de l'activité de résumer. Cette superposition est rendue nécessaire par la particularité du texte, dont la réputation est de déjouer sans cesse les attentes du lecteur. On voit à nouveau l'importance de l'objet, ici le texte contemporain réputé «bizarre », pour comprendre les différentes formes de sédimentation. Ce sont les propriétés réactives des deux textes qui, dans l'analyse, permettent de contraster l'activité de condensation selon qu'elle s'exerce sur un texte réputé littéraire, dont la réputation rend évident le sens de l'histoire, ou sur un texte réputé difficile qui ne permet pas le passage rassurant et tranquille (scolaire serions-nous tenté d'écrire) du sens de premier degré vers le sens de second degré, de la surface contraignante du texte à la production d'une interprétation.

\section{Thèse 6 : la disciplinarisation : l'une des causes de la sédimentation des pratiques}

Si la sédimentation s'observe en synchronie dans toute pratique d'enseignement, au niveau individuel de chaque acteur, sous des formes différentes, elle est aussi le résultat d'un processus de disciplinarisation caractéristique de la forme école moderne qui se met en place au XIX ${ }^{e}$ siècle. Ce processus est l'une des formes que prend la transposition didactique externe. Sans nier les différences entre les degrés primaire et secondaire, on observe qu'un même terme s'impose progressivement pour désigner l'organisation des savoirs dans l'ensemble du système scolaire, terme qui diffère en fonction des langues : « discipline » en français, "Schulfach» en allemand, "school subject » en anglais, 
"disciplina » en italien, etc. Une même unité s'impose peu à peu comme " mesure " commune pour concevoir la distribution des contenus d'enseignement. En effet, la gestion des classes et des degrés, qui font désormais système, nécessite des outils analogues pour la conception globale des curriculums: l'allocation des ressources temporelles et humaines, et, partant, la reconnaissance même de ces disciplines.

La disciplinarisation, définie comme l'organisation des savoirs en disciplines scolaires, n'est pas le produit d'un processus, fixé une fois pour toutes. Les disciplines émergent, se déploient, s'imposent, se regroupent, s'amalgament voire disparaissent, par différenciation, fission, extension ou fusion. Au sein d'une même discipline, des contenus émergent, se déploient, s'imposent, etc., et sont sans cesse ré-agencés et redéfinis. Certaines disciplines se scindent en plusieurs disciplines (sciences), d'autres s'ajoutent (langues étrangères au primaire), d'autres encore suivent un processus de différenciation interne (mathématiques), tandis que certaines disparaissent dans la plupart des systèmes scolaires (catéchisme, travaux manuels). Le fait même que les disciplines organisent les savoirs selon des principes comparables dans les différents niveaux et degrés scolaires permet aussi de faire passer des contenus d'un niveau ou d'un type d'école à un autre, processus qu'on peut historiquement observer en mathématiques (D'Enfert, 2005), en sciences (Lebeaume, 2008), en histoire (Falaize, 2016) ou en littérature (Schneuwly, 2016). Cette observation vaut également pour la lecture. Les changements opérés dans l'organisation de la discipline «français » ont pour effet contradictoire que tendanciellement tout est enseigné à tous dans le sens où l'enseignement se focalise partout sur la thématique et l'interprétation de textes ou les connaissances de la langue. En même temps, subsiste une modalité plus ancienne, classique, de structuration différenciée des savoirs « réservés » en fonction des niveaux entre cycle moyen et cycle d'orientation (collège): une sédimentation de strates historiques (Thévenaz et al., 2011).

Sans cesse reconfigurées, les disciplines scolaires sont l'œuvre d'acteurs multiples. Mais elles sont en premier lieu le produit de l'agir des enseignants eux-mêmes. Ces derniers en discutent les contenus et méthodes, aussi bien dans des échanges informels que publiquement, dans leurs associations, les revues, les congrès. Surtout, ils les produisent quotidiennement dans leur travail avec les élèves. On peut dès lors concevoir ces disciplines comme un cadre de pensée et d'action pour les enseignants, mais aussi pour les élèves, ou, pour l'exprimer autrement, comme des « formes d'agir et de penser sédimentées au cours du temps et, en tant que telles, durables et résistantes au changement " (Mainer Bauqué, 2010, p. 24). Ce cadre ou ces formes se reproduisent et se transforment continuellement par la pensée, le discours et l'action des protagonistes en présence, enseignants et élèves, inscrits dans leur contexte d'énonciation plus global (et leurs dimensions socioculturelles et politicoéconomiques).

29 À partir d'une analyse approfondie des plans d'études et manuels en usage en Suisse romande depuis une centaine d'années ${ }^{17}$, nous avons décrit l'évolution de l'organisation des savoirs dans la discipline « français » sous forme d'une double tendance :

(i) l'instauration d'une progression plus spiralaire

(ii) la sédimentation de pratiques qui perpétuent des éléments d'une progression linéaire.

Les deux voies relativement étanches entre primaire et secondaire se mêlent de plus en plus par un processus de transformation du principe même de construction 
curriculaire qui devient davantage spiralaire. La littérature devient objet d'enseignement à tous les degrés de la scolarité. Ceci se réalise surtout par le fait que des textes réputés littéraires deviennent potentiellement objet d'études au niveau de l'école primaire déjà ; ils ne sont (seulement) plus des " pré-textes » pour des activités de grammaire ou de vocabulaire et des modèles pour apprendre à écrire, comme ce fut le cas dans le dispositif didactique classique plus ancien (Reuter, 1996), mais sont travaillés du point de vue de la compréhension et de l'interprétation. Sur l'ensemble des corpus rassemblés depuis vingt ans, on constate des pratiques innovantes à tous les degrés de l'école primaire et secondaire, qui se greffent sur les démarches déjà là. $A u$ secondaire II, cependant, même si des pratiques innovantes sont observées, l'approche du texte-prétexte, caractéristiques des approches établies dès la fin du $19^{\mathrm{e}}$ siècle, est encore largement répandue.

\section{Thèse 7 : une discipline scolaire sert à discipliner...}

La disciplinarisation comme organisation des savoirs sous formes de disciplines a une fonction précise : "Une "discipline", c'est aussi, pour nous, en quelque domaine qu'on la trouve, une façon de discipliner l'esprit, c'est-à-dire de lui donner des méthodes et des règles pour aborder les différents domaines de la pensée, de la connaissance et de l'art» (Chervel, 1988, p. 64). Pour le dire en d'autres termes, discipliner signifie faire acquérir les objets d'enseignement et d'apprentissage propres à la discipline, afin de transformer les modes de penser, parler et faire. Les moyens de cette transformation sont les savoirs et pratiques systématiquement organisés et la systématicité de leur enseignement, visant à construire un rapport plus conscient et volontaire à ses propres processus psychiques. Prenons l'exemple de l'enseignement de la proposition et de la phrase en grammaire que nous empruntons à L. S. Vygotskij. Il montre que le savoir grammatical ne fonctionne pas comme auxiliaire à une autre action ou à la pensée ; il n'est pas abordé seulement pour son utilité externe. Le savoir grammatical lui-même est une condition de la transformation du rapport aux processus propres et aux savoirs déjà là. En quoi cet enseignement transforme-t-il les processus psychiques ? Il les transforme précisément parce que les savoirs sur le fonctionnement de la phrase permettent d'avoir un rapport plus conscient et plus volontaire par rapport à certaines dimensions du parler et de l'écrire. Les savoirs transmis par l'enseignement de la grammaire sont des instruments de transformation du rapport à son propre langage.

On peut, pour réfléchir sur la disciplination, distinguer trois points de vue inextricablement liés :

(i) celui de l'organisation des savoirs prescrits par le système scolaire, fruit de la transposition didactique externe, médiatisée par le processus de disciplinarisation qui est la forme de transposition dans le cadre de la forme école moderne ;

(ii) celui des pratiques et instruments des enseignants agissant à l'intérieur de la discipline en abordant un objet d'enseignement donné ; et

(iii) celui de la transformation des modes de penser, dire et faire des élèves dans leur progression à travers le système. Le rapport entre les deux derniers est au cœur des réflexions vygotskiennes dans toute une série de textes (voir pour une vue d'ensemble Mangott, 1995).

33 " Nous avons enseigné à l'enfant le pfennig, et il s'est développé pour le mark. Un pas dans l'enseignement peut signifier 100 pas dans le développement » écrit L. S. Vygotskij 
(1985 [1934], p. 253), pointant à la fois l'interdépendance et l'indépendance des processus d'enseignement et d'apprentissage et développement.

Quand on enseigne la littérature, on discipline en fait les élèves pour qu'ils adoptent un nouveau rapport aux textes (voir dans le présent numéro le texte de Vuillet et Védrines). Les élèves apprennent par la médiation de toute une série de dispositifs à réaliser sur les textes, lesquels dispositifs instaurent précisément ce texte en tant que texte littéraire. C'est ce que nous avons appelé la "réputation littéraire ». Les différentes formes du dispositif résumer, dont nous avons analysé plus haut le processus de sédimentation dans la thèse 5 représente cette idée, introduite plus haut dans la thèse 4, qu'un dispositif didactique est à la fois un instrument que l'enseignant met en œuvre et que les élèves s'approprient en le transformant au cours de leur scolarité, transformant leur propre processus psychique (Ronveaux, 2018). L'observation de l'activité de résumé dans les différents degrés scolaires atteste d'une disciplination:

(i) les élèves du primaire résument et analysent un texte au niveau de la compréhension de la diégèse (il s'agit de montrer qu'on a compris l'histoire);

(ii) les élèves lecteurs du secondaire I sont invités à résumer « objectivement » une histoire, mais prennent déjà de la distance, en travaillant sur le point de vue, les figures du discours, la versification, etc. ;

(iii) au secondaire II, les élèves deviennent des lecteurs méthodiques, qui ont intériorisé les instruments stylistiques, rhétoriques, génériques, pour établir la réputation littéraire des textes et peuvent les appliquer à tout texte. Résumer, à ce degré, a une tout autre fonction : il sert davantage à mettre d'accord un collectif sur ce qu'on va interpréter.

Le changement de la fonction du dispositif « résumer » est un des multiples indices qui montrent le processus de disciplination. Changement de dispositifs et transformation profonde des productions des élèves qui nous incite à parler de rupture. Et en même temps, à tous les niveaux, y compris déjà au primaire, certes très timidement, se crée, par le résumé, un rapport au texte en tant que texte, résumé à partir duquel on s'ajuste dans le cadre de la classe à partir de la formulation d'une base sémantique commune avant d'interpréter le texte. Il y a donc, dans ce processus de disciplination, continuité et rupture tout à la fois. Continuité parce que ce même instrument est déjà présent à tous les premiers degrés et comprend en germe les potentialités qui vont se développer tout au long du curriculum. Rupture parce que l'activité de condensation se décline en instruments distincts produisant la transformation des élèves.

C'est sans doute l'un des résultats les plus intéressants réalisés dans nos recherches empiriques concernant la disciplination : la description, à partir de multiples indicateurs, de la construction d'un nouveau rapport, littéraire, au texte, lequel rapport s'apprend très tôt, dès l'école primaire et se développe au fur et à mesure de la scolarité dans la continuité et la rupture. Le concept de disciplination montre aussi que, dans la pratique des enseignants, on enseigne les mêmes choses dans les différents degrés, mais de manières différentes. Peut-il en être autrement, puisque l'élève n'est plus le même? Il a transformé fondamentalement son rapport au texte. C'est cette transformation par la rupture qui constitue le développement dans sa continuité. 


\section{Éléments de conclusion} bref recul épistémologique pour ouvrir de nouvelles questions. L'approche instrumentale, nous l'avons montré, est fondée notamment sur la conception de L. S. Vygotskij ${ }^{18}$ du rapport entre enseignement et développement et le potentiel qu'il décèle dans ce rapport fondamentalement et nécessairement contradictoire pour le développement et que nous pensons pouvoir traduire dans l'effet de disciplination qui est loin d'être un effet de surface, mais qui affecte profondément, de manière contradictoire, le développement de la personnalité. Ce rapport se réalise à travers deux formes de médiation: celle qu'effectue l'enseignant par le processus de double sémiotisation qui offrent des outils sémiotiques que les élèves peuvent s'approprier pour transformer leurs propres processus psychiques. Dans cette conception, nous l'avons montré, les disciplines scolaires, fruit de la disciplinarisation comme l'une des dimensions de la transposition didactique, jouent une rôle médiateur central dans le processus de disciplination conçu comme développement de la personne dans une perspective discutée dans le concept de la Bildung allemand que L. S. Vygotskij invoque en se référant notamment à J.F. Herbart (Schneuwly, 2018).

ent, et nous revenons ici aux possibles différences, voire contradictions théoriques, entre l'approche instrumentale et les travaux se développant dans la lignée de la théorie anthropologique de la didactique, de la théorie des situations ou encore des travaux principaux dans la théorie de l'action conjointe pour nommer seulement des courants représentatifs qui se sont développés en lien avec les concepts fondateurs d'Y. Chevallard à propos de la transposition didactique. Elles nous interrogent puissamment. On peut dire que l'une des dimensions de nos travaux consiste à montrer les potentialités d'un système en permanente réforme et adaptation dans une lignée qu'on pourrait décrire, en simplifiant beaucoup, comme étant celle de ComeniusHumboldt/ Condorcet-Vygotskij, tout en mettant en évidence, nous l'avons mentionné, les effets de distinction. Cette autre tradition de pensée dévoile et insiste sur les effets pernicieux de "temps, textualisation et contrat didactique classique", inauguré précisément par Comenius. Ce dispositif classique, la «forme scolaire » classique, a pour effet la « déconcertation cognitive " (Chevallard, 1991), la "monumentalisation » (ibid., 2004), un "maître sans mémoire " (Brousseau et Centeno, 1991, p. 182). Il s'agit donc de la transformer radicalement, de la soumettre à une triple "révolution adidactique, épistémologique et praxéologique »(Sensevy, 2011). De ce point de vue, l'approche instrumentale que nous avons développée pourrait être lue comme incarnant le " temps didactique classique ", voire comme étant son apologie. D'un autre côté, l'analyse de la sédimentation incessante montre que, dans la forme école, agissent sans cesse des tendances contradictoires qu'il s'agit d'analyser en détail, si l'on veut rendre compte de la transposition didactique interne dans sa dynamique constitutive. 
Questions générales largement ouvertes au débat donc, que nous mènerons à d'autres occasions.

\section{BIBLIOGRAPHIE}

AEBY DAGHÉ, s. (2010). « Quels gestes professionnels pour quelles activités scolaires ? Lire et interpréter des textes littéraires ", Repères. Recherches en didactique du français langue maternelle 42, p. 127-144. En ligne : https://doi.org/10.4000/reperes.255

AEBY DAGHÉ, S. (2014). Candide, la fée carabine et les autres. Vers un modèle didactique de la lecture littéraire. Bruxelles : Peter Lang.

AEBY DAGHÉ, S. \& SCHNEUWLY, B. (2018). « Des dispositifs pour enseigner ». In : Ronveaux, C. \& Schneuwly, B. (éds), Lire des textes réputés littéraires : disciplination et sédimentation. Enquête au fil des degrés scolaires en Suisse romande. Bruxelles : Peter Lang, p. 241-255.

ALTHUSSER, L. (1976). « Idéologie et appareils idéologiques d'État ». In : Positions. Paris : Éditions sociales, p. 67-126. En ligne : http://classiques.uqac.ca/contemporains/althusser_louis/ ideologie_et_AIE/ideologie_et_AIE.html

BAIN D. et al. (1985). Contributions à la pédagogie du texte. Genève : Université de Genève, Faculté de psychologie et des sciences de l'éducation. En ligne : https://archive-ouverte.unige.ch/unige: 34469.

BAIN D. et al. (1988). Contributions à la pédagogie du texte II. Genève : Section des sciences de l'éducation, Université de Genève. En ligne : https://archive-ouverte.unige.ch/unige:34218.

BELHADJIN, A. \& PERRET, L. (éds) (2020). L'Extrait et la fabrique de la littérature scolaire. Bruxelles : Peter Lang

BERNIÉ, J.-P., BROSSARD, M. (éds) (2013). Vygotski et l'école. Apports et limites d'un modèle théorique pour penser l'éducation et la formation. Pessac: Presses universitaires de Bordeaux.

BRECHT, B. (1979) [1968], Me-Ti. Livre des retournements, Paris, L'arche.

BRONCKART, J. (2017). « Théories linguistiques et psychologie du développement. F. de Saussure et V. Vološinov en appui aux thèses interactionnistes ». Cahiers de l'ILSL 52, p. 5-25. En ligne : https://archive-ouverte.unige.ch/unige:109876.

BRONCKART, J.-P. \& PLAZAOLA GIGER, I. (1998). « La transposition didactique. Histoire et perspectives d'une problématique fondatrice ». Pratiques 97-98, p. 35-58. En ligne : https://doi.org/10.3406/ prati.1998.2480²

BROUSSEAU, G (2011). « La théorie des situations didactiques en mathématiques. » Éducation \& Didactique 5-1, p. 101-104. En ligne : https://doi.org/10.4000/educationdidactique.1005 BROUSSEAU, G, \& CENTENO, J. (1991). « Rôle de la mémoire didactique de l'enseignant ». Recherches en Didactique des Mathématiques 11, p. 167-210.

CHERVEL, A. (1988). « Histoire des disciplines scolaires ». Histoire de l'éducation 38, p. 59-119. En ligne : https://doi.org/10.3406/hedu.1988.1593 
CHERVEL, A. (2006). Histoire de l'enseignement du français $d u X V I I^{e} a u X X^{e}$. Paris : Retz.

CHEVALLARD, Y. (1985). La Transposition didactique : du savoir savant au savoir enseigné. Grenoble : Éd.

La Pensée Sauvage.

CHEVALLARD, Y. (1991). « Sur la déconcertation cognitive. ». Interactions didactiques 12, p. 27-51.

CHEVALLARD, Y. (1992). « Concepts fondamentaux de la didactique : perspectives apportées par une approche anthropologique ». Recherches en Didactique des Mathématiques 12, p. 73-112.

CHEVALLARD, Y. (2004). « Vers une didactique de la codisciplinarité. Notes sur une nouvelle épistémologie scolaire ». Journées de L'Association pour les Recherches Comparatistes en Didactique, Lyon, 3-4 mai.

CHEVALLARD, Y., \& BOSCH, M. (2020). « Didactic Transposition in Mathematics Education ». In : Lerman, S. (éd.), Encyclopedia of Mathematics Education. New York : Springer International Publishing, p. 214-218.

COMENIUS, J. A. (2005) [1648]. Novissima linguarum methodus. Trad du latin par H. Jean. Genève: Droz. CORDEIRO, G., RONVEAUX, C. \& GRAFE (2009). « Recueil et traitement des données ». In : Schneuwly, B. \& Dolz, J. (éds), Des objets enseignés en classe de français. Le travail de l'enseignant sur la rédaction de textes argumentatifs et sur la subordonnée relative. Rennes : Presses universitaires de Rennes, p. 83-100.

DARME-XU, A. (2019). Enseigner la grammaire pour développer l'expression de la pensée : éléments d'histoire de la grammaire scolaire en Suisse romande (1830-1990). Thèse en sciences de l'éducation : Université de Genève.

DAUNAY, B. (2016). « Quelques réflexions sur le sujet d'une description didactique ». Éducation \& Didactique 10-2, p. 123-136. En ligne : https://doi.org/10.4000/educationdidactique.2488 D'ENFERT, R. (2005). « L'enseignement mathématique dans le primaire et le secondaire au début du $\mathrm{XX}^{\mathrm{e}}$ siècle. Vers une culture commune ? ». In : Caspard, P., Luc, J.-N. \& Savoie, P. (éds.), Lycées, lycéens, lycéennes, deux siècles d'histoire. Lyon : INRP, p. 247-255.

DENIZOT, N. (2018). La Culture scolaire : perspectives didactiques. Habilitation à diriger des recherches : Université de Lille.

DENIZOT, N. \& RONVEAUX, C. (éds) (2019). La Lettre enseignée. Perspective historique et comparaison européenne. Grenoble : Université Grenoble Alpes Éditions.

DOLCH, J. (1982) [1959]. Lehrplan des Abendlandes. Zweieinhalbtausend Jahre seiner Geschichte. Darmstadt : Wissenschaftliche Buchgemeinschaft.

FALAIZE, B. (2016). L'Histoire à l'école élémentaire depuis 1945. Rennes : Presses universitaires de Rennes.

FRANCK, O. (2017). À la recherche de l'archiélève lecteur à travers l'analyse du geste de planification. Rôle des élèves dans les modifications de séquences d'enseignement. Thèse de doctorat en sciences de l'éducation : Université de Genève. En ligne : https://archive-ouverte.unige.ch/unige:92154.

FRANCK, O. (2018). « Modifications des plans d'enseignement : un espace d'invention pour enseignants et élèves ». In : Ronveaux, C., \& Schneuwly, B. (éds), Lire des textes réputés littéraires : disciplination et sédimentation. Enquête au fil des degrés scolaires en Suisse romande. Bruxelles : Peter Lang, p. 195-212.

GABATHULER, C. (2016). Apprécier la littérature. La relation esthétique dans l'enseignement de la lecture de textes littéraires. Rennes: Presses universitaires de Rennes. 
JAUBERT, M., \& REBIÈRE, M. (2019). « Le scénario langagier didactique, un outil dans le processus de construction des savoirs ? Un exemple : l'enseignement et l'apprentissage de la lecture ». Raisons éducatives 23, p. 153-176. En ligne : https://doi.org/10.3917/raised.023.0153

LEBEAUME, J. (2008). L'Enseignement des sciences à l'école. Des leçons de choses à la technologie. Paris : Delgrave.

LOUICHON, B., BISHOP, M.-F. \& RONVEAUX, C. (éds) (2017). Les Fables à l'école. Un genre patrimonial européen?. Bruxelles : Peter Lang.

MAINER BAUQUÉ, J. (2010). « La historia de las disciplinas escolares. Génesis y problemas de un joven campo de investigación ». Seminario del Doctorado interinstitucional de Educación de la Universidad Distrital José Caldâs : Bogotâ, 10 août.

MANGOTT, D. (1995). Kontinuität und Wandel im Schaffensprozess von LS Vygotskij: Ein russischdeutsches Lexikon zur Ideengeschichte 1926-1934. Thèse en lettres : Université d'Innsbruck.

MARX, K. (1953). Grundrisse der Kritik der politischen Ökonomie. Berlin : Dietz.

MONNIER-SILVA, A.-C. (2018). Le Temps des dissertations. Chronique de l'accès des jeunes filles aux études supérieures (Genève, $\mathrm{XIX}^{e}-\mathrm{XX} \mathrm{X}^{\mathrm{e}}$ ). Genève : Droz

REUTER, Y. (1996). « Éléments de réflexion sur la place et les fonctions de la littérature dans la didactique du français à l'école ». Repères. Recherches en didactique du français langue maternelle 13, p. 7-25. En ligne : https://doi.org/10.3406/reper.1996.2172

RIONDET, X. (2020). "Changer l'éducation, changer la société ». Contre-histoires à destination des futurs enseignants. Habilitation à diriger des recherches : Université de Nancy.

ROCHEX, J.-Y., JOIGNEAUX, C. \& NETER, J. (éds.) (2018). Histoire, culture, développement : questions théoriques, recherches empiriques. Actes du $6^{e}$ séminaire international Vygotski. Paris : Université Paris 8 Saint Denis/ CRTD/Cnam. En ligne : https://www.unige.ch/SIV2018/files/3315/3251/3499/ Actes_SIV_6_Corr.28juin18.pdf. RONVEAUX, C. (2018). « Résumer, comprendre et interpréter une histoire ». In : Ronveaux, C., \& Schneuwly, B. (éds), Lire des textes réputés littéraires : disciplination et sédimentation. Enquête au fil des degrés scolaires en Suisse romande. Bruxelles : Peter Lang, p. 257-277.

RONVEAUX, C., \& SCHNEUWLY, B. (éds) (2018). Lire des textes réputés littéraires : disciplination et sédimentation. Enquête au fil des degrés scolaires en Suisse romande. Bruxelles : Peter Lang. SCHNEUWly, B. (1995). « De l'utilité de la "transposition didactique” ». In : Chiss, J.-L., David, J., \& Reuter, Y. (éds), Didactique du français. Paris : Nathan, p. 47-62.

SCHNEUWLY, B. (2009). « Le travail enseignant ». In : Schneuwly, B. \& Dolz, J. (éds), Des objets enseignés en classe de français. Le travail de l'enseignant sur la rédaction de textes argumentatifs et sur la subordonnée relative. Rennes : Presses universitaires de Rennes, p. 29-43.

SCHNEUWLY, B. (2016). «Forme-t-on au même rapport à la langue des deux côtés de la Sarine? Premières explorations dans les livres de lecture durant un siècle ». Forum Lecture, 2. En ligne : https://www.forumlecture.ch/sysModules/obxLeseforum/Artikel/573/2016_2_Schneuwly.pdf SCHNEUWLY, B. (2018). «Schulfächer: Vermittlungsinstanzen von Bildung ». Zeitschrift für Erziehungswissenschaft, 21, 279-298.

SCHNEUWLY, B., \& BRONCKART, J.-P. (éds) (1985). Vygotsky aujourd'hui. Neuchâtel/Paris : Delachaux et Niestlé. 
SCHNEUWLY, B. \& DOLZ, J. (2009). Des objets enseignés en classe de français. Le travail de l'enseignant sur la rédaction de textes argumentatifs et sur la subordonnée relative. Rennes: Presses universitaires de Rennes.

SENSEVY, G. (2011). Le Sens du savoir. Éléments pour une théorie de l'action conjointe en didactique. Bruxelles : De Boeck.

SÈVE, L. (1974). Marxisme et théorie de la personnalité. Paris : Éditions sociales.

SÈVE, L. (2008). « L'homme » ? Paris : La Dispute.

SIMONDON, G. (1969). Du mode d'existences des objets techniques. Paris : Aubier.

THÉVENAZ-CHRISTEN, T. (2014). La lecture enseignée au fil de l'école obligatoire. L'exemple genevois. Namur : Presses universitaires de Namur.

THÉVENAZ-CHRISTEN, T. et al. (2011). « Progression : un concept fondateur de la didactique. L'exemple de la lecture à travers l'école obligatoire ». In : Daunay, B., Reuter, Y. \& Schneuwly, B. (éds), Les Concepts et les méthodes en didactique du français. Namur : Presses universitaires de Namur, p. 85-115.

TIBERGHIEN, A. \& SENSEVY, G. (2015). « Transposition didactique ». In : Gunstone, R. (éd.), Encyclopedia of Science Education. Dordrecht : Springer, p. 1083-1086.

TINEMBART, S. (2015). Le Manuel scolaire de français, entre production locale et fabrique de savoirs. Le cas des manuels et de leurs concepteurs dans le canton de Vaud au $19^{e}$ siècle. Thèse en sciences de l'éducation : Université de Genève.

TOULOU, S. (2008). Devenir griot professionnel : éducation formelle ou informelle? Analyse des enseignements langagiers dans la perspective de la transposition didactique. Thèse en sciences de l'éducation : Université de Genève.

VÉDRINES, B. (2017). L'Assujettissement littéraire. Thèse en sciences de l'éducation : Université de Genève.

VYGoTSKIJ, L. S. (2014) [1930]. « La méthode instrumentale en psychologie ». In : Histoire du développement des fonctions psychiques supérieures. Paris : La Dispute, p. 565-574.

VYGOTSKIJ, L. S. (2014) [1928-30]. Histoire du développement des fonctions psychiques supérieures. Paris : La Dispute.

VYGOTSKIJ, L. S. (1985) [1934]. Pensée et langage. Paris : Éditions sociales.

WIRTHNER, M. (2017). Outils d'enseignement : au-delà de la baguette magique. Outils transformateurs, outils transformés dans des séquences d'enseignement en production écrite. Berne : Peter Lang.

\section{NOTES}

1. Ce texte a paru dans le numéro 97-98 de la revue Pratiques, entièrement dédié à la question de la place du concept de transposition didactique en didactique du français.

2. Constatant la multiplicité des translittérations de ce nom, nous utilisons dorénavant celle, officielle, de la norme ISO 9.

3. Nous ne traitons pas ici les apports décisifs dans notre travail, du côté de la linguistique, de la théorie du texte et du discours, plus particulièrement de apports de F. de Saussure et de V. Voloshinov (anciennement M. Bakhtine) (Bronckart, 2017). 
4. Nous sommes loin d'être les seuls à nous baser sur la théorie de L. S. Vygotskij pour analyser et comprendre le fonctionnement de l'enseignement et du développement. Mentionnons notamment les travaux menés dans l'équipe jadis dirigée par M. Brossard et J.-P. Bernié (2013) qui se poursuivent aujourd'hui (Jaubert \& Rebière, 2019); ou dans l'équipe Escol qui a édité les Actes du $6^{e}$ Séminaire International Vygotskij (Rochex, Joigneaux \& Netter, 2018).

5. Pensons aux influences indéniables de la théorie de L. Althusser dans la première formulation de la théorie dans l'ouvrage d'Y. Chevallard (1985) Transposition didactique, notamment de la théorie des appareils idéologiques d'État et de l'interpellation du sujet (Althusser, 1976; pour une analyse plus générale de l'influence du groupe "École » d'Althusser sur la pensée éducative en France, en particulier C. Baudelot, R. Establet, R. Balibar et M. Tort, voir Riondet, 2020 ; ce groupe comprenait aussi pendant un certain temps, M. Verret, qui, comme on le sait, est à l'origine du concept de transposition didactique). On trouve des traces de cette influence dans la manière d'aborder la question des élèves et le peu d'attention accordée à la question du développement. Et pensons de l'autre côté au fait, non dénué de signification, que c'est L. Sève (voir 1974 ; 2008) qui a initié pour l'essentiel les traductions de L. S. Vygotskij en français, lui qui combattait l'antihumanisme théorique de L. Althusser en proposant une théorie de la personnalité dans laquelle la question du développement et de la biographie sont centrales.

6. La littérature consacrée à ce concept est immense et il serait vain de l'énumérer et encore moins de la discuter ici. Rappelons, dans le cadre de cette revue, le numéro spécial 97-98 La transposition didactique en français. Nous nous fondons plus particulièrement sur l'ouvrage inaugural d'Y. Chevallard (1985). Pour des présentations récentes, simples, condensées, voir A. Tiberghien \& G. Sensevy (2015) et Y. Chevallard \& M. Bosch (2020).

7. Nous reprenons ici la terminologie d'Y. Chevallard (1985, p. 39) qui propose le schéma " objet de savoir $\rightarrow$ objet à enseigner - objet d'enseignement ». Nous utilisons ce dernier terme pour désigner à la fois l'objet à enseigner et l'objet enseigné.

8. Pour une histoire très exhaustive de ces multiples formes depuis plus de 3000 ans, voir J. Dolch (1982 [1959]) ; pour l'analyse de l'organisation progressive dans la pratique de formation de griots, voir S. Toulou (2008).

9. La théorie des situations didactiques de G. Brousseau (2011) permet particulièrement bien de modéliser cette réalité.

10. Dans ce texte comme dans les autres, nous allons utiliser les termes sans distinction systématique, en fonction du contexte et des références mobilisées (par exemple chez L. S. Vygotskij, «acte instrumental » d'une part et «outil» de l'autre). La distinction systématique proposée par G.Simondon (1969) - outil médiateur de l'action, instrument médiateur de sens - n'est pas opérationnelle dans le contexte des outils/ instruments sémiotiques.

11. Nous empruntons cette formule à J. A. Comenius (2005 [1648], p. 159) qui définit le scire, le savoir, en ces termes "aliquid effigiare posse: seu mente, seu manu, seu lingua. » [donner forme à quelque chose soit par l'esprit, soit par la main, soit par la langue ; notre traduction], précisant que le premier mode est de l'ordre du "Wissen" [knowledge], les deux autres de l'ordre du "Können" [know how]. 
12. L.S. Vygotskij utilise le terme d'« outils psychologiques» (« психолщгическоЕ орудиЕ ») pour décrire ce que sont les signes; ils ont une fonction instrumentale, sa méthode étant une "méthode instrumentale ". Très régulièrement par ailleurs, il va parler du mot comme outil, ce que nous avons généralisé par les termes d'« outils sémiotiques ». C'est la raison pour laquelle nous parlons d'une approche instrumentale (de la transposition didactique).

13. Nous laissons ici de côté la question de la planification (voir Franck, 2018).

14. Nous n'allons pas ici développer la problématique de l'enregistrement - en soi déjà un point de vue - de la transcription ou du synopsis, tout comme celle des analyses que cela rend possible. Contentons-nous de dire combien nous sommes d'accord avec cette formulation de B. Daunay (2016, p.132) : « décrire, c'est éviter de décrier, c'est dire sans maudire ; mais aussi sans prédire, ni prescrire ».

15. La métaphore géologique ne devrait pas induire dans l'erreur d'un figement immuables de couches comme le montre d'ailleurs le discours lui-même décrivant combien les couches s'entremêlent, surgissent, disparaissent.

16. Toutes nos analyses des pratiques comprennent une description de l'histoire de l'enseignement des objets soumis à l'analyse. Par ailleurs, nous avons mené plusieurs projets de recherche parallèles sur l'histoire de l'enseignement (voir note 17).

17. Voir notamment les travaux publiés par Grafelect (Thévenaz-Christen, 2014), par HELICE (Louichon, Bishop \& Ronveaux, 2017; Belhadjin \& Perret, 2020; Denizot \& Ronveaux, 2019), ou encore les travaux réalisés dans le cadre du projet «Transformations des savoirs scolaires en suisse 1830-1990" (FNS CRSII1_141826) (Tinembart, 2015 ; Monnier-Silva, 2018; Darme Xu, 2019), ou dans le cadre d'autres thèses de doctorat (Gabathuler, 2016 ; Védrines, 2017).

18. Si l'on peut en effet dire que J. Piaget défend un « constructivisme radical » comme le notent G. Brousseau (2011) et, à sa suite, G. Sensevy (2011), L. S. Vygotskij se situe, lui, aux antipodes de cette position, critiquant même au passage les effets néfastes que produit l'introduction massive d'une approche basée sur une telle conception de l'école.

\section{RÉSUMÉS}

Cette contribution présente une définition de la transposition didactique et un système conceptuel s'y rattachant forgé par notre équipe de recherche tout au long d'un programme de recherche de trente années. L'exposé des sept thèses suit l'ordre d'apparition des concepts qui se sont imposés au moment de formuler les questions de recherche et leur mise à l'épreuve de l'empirie. Nous définissons successivement: la transposition didactique comme objet central de la didactique (thèse 1), les outils sémiotiques du travail de l'enseignant (thèse 2), la séquence d'enseignement comme unité du travail de l'enseignant (thèse 3) et sa panoplie d'instruments (thèse 4), la sédimentation des pratiques (thèse 5), la disciplinarisation (thèse 6) et la discipline scolaire comme source de disciplination (thèse 7). Chacune de ces thèses est illustrée par quelques-uns des résultats des recherches empiriques menées dans l'équipe de recherche. 
This contribution presents a definition of didactic transposition and the conceptual system attached to it that our research team has developed over a thirty-year research program. The presentation of the seven theses follows the order of emerging concepts when formulating the research questions and their empirical testing. We successively define didactic transposition as the central object of didactics (thesis 1), the semiotic tools of the teacher's work (thesis 2), the teaching sequence as a unit of the work of the teacher (thesis 3 ) and its panoplies of instruments (thesis 4), the sedimentation of practices (thesis 5), disciplinarization (thesis 6) and school discipline as a source of disciplination (thesis 7). Each of these theses is illustrated by some of the results of the empirical research of our research team.

INDEX

Keywords : Didactic transposition, instrumental approach, teaching, development, French subject matter

Mots-clés : Transposition didactique, approche instrumentale, enseignement, développement, discipline français.

\section{AUTEURS}

\section{BERNARD SCHNEUWLY}

Université de Genève, Grafe, $\mathrm{CH}-1205$ Genève, Suisse

\section{CHRISTOPHE RONVEAUX}

Université de Genève, Grafe, $\mathrm{CH}-1205$ Genève, Suisse 